\title{
Alternative splicing and nonsense-mediated decay regulate telomerase reverse transcriptase (TERT) expression during virus-induced lymphomagenesis in vivo
}

Souheila Amor ${ }^{1}$, Sylvie Remy ${ }^{1,2}$, Ginette Dambrine ${ }^{1,2}$, Yves Le Vern ${ }^{2,3}$, Denis Rasschaert ${ }^{1 *}$, Sylvie Laurent ${ }^{1,2}$

\begin{abstract}
Background: Telomerase activation, a critical step in cell immortalization and oncogenesis, is partly regulated by alternative splicing. In this study, we aimed to use the Marek's disease virus (MDV) T-cell lymphoma model to evaluate TERT regulation by splicing during lymphomagenesis in vivo, from the start point to tumor establishment.

Results: We first screened cDNA libraries from the chicken MDV lymphoma-derived MSB-1 T- cell line, which we compared with B (DT40) and hepatocyte (LMH) cell lines. The chTERT splicing pattern was cell line-specific, despite similar high levels of telomerase activity. We identified 27 alternative transcripts of chicken TERT (chTERT). Five were in-frame alternative transcripts without in vitro telomerase activity in the presence of viral or chicken telomerase RNA (vTR or chTR), unlike the full-length transcript. Nineteen of the 22 transcripts with a premature termination codon (PTC) harbored a PTC more than 50 nucleotides upstream from the $3^{\prime}$ splice junction, and were therefore predicted targets for nonsense-mediated decay (NMD). The major PTC-containing alternatively spliced form identified in MSB1 (ie10) was targeted to the NMD pathway, as demonstrated by UPF1 silencing. We then studied three splicing events separately, and the balance between in-frame alternative splice variants ( $\mathrm{d} 5 \mathrm{f}$ and $\mathrm{d} 10 \mathrm{f}$ ) plus the NMD target i10ec and constitutively spliced chTERT transcripts during lymphomagenesis induced by MDV indicated that basal telomerase activity in normal T cells was associated with a high proportion of in-frame non functional isoforms and a low proportion of constitutively spliced chTERT. Telomerase upregulation depended on an increase in active constitutively spliced chTERT levels and coincided with a switch in alternative splicing from an in-frame variant to NMD-targeted variants.
\end{abstract}

Conclusions: TERT regulation by splicing plays a key role in telomerase upregulation during lymphomagenesis, through the sophisticated control of constitutive and alternative splicing. Using the MDV T-cell lymphoma model, we identified a chTERT splice variant as a new NMD target.

\section{Background}

The telomeric enzyme complex, consisting of a telomerase reverse transcriptase (TERT) and an RNA template (TR), adds terminal telomeric repeats to the end of the chromosome, to maintain telomere length during cell proliferation [1,2]. Most normal somatic cells lack telomerase activity, whereas telomerase activation is observed in proliferating cells and cancer cells $[3,4]$.

\footnotetext{
* Correspondence: denis.rasschaert@univ-tours.fr

'Equipe TLVI, Université François Rabelais de Tours, UFR Sciences et

Techniques, Parc de Grandmont 37200 Tours France

Full list of author information is available at the end of the article
}

Telomerase activity is highly regulated in lymphocytes, being expressed only in activated lymphocytes [5]. In humans, the hTR transcript is constitutively produced, whereas the production of hTERT is highly regulated at both the transcriptional and post-transcriptional levels $[6,7]$. Alternative splicing of the hTERT transcript plays a role in this regulation and 10 alternatively spliced sites have been identified in the hTERT gene [8]. Notably, the alpha isoform, corresponding to an in-frame deletion in the RT motif, appears to be a dominant inhibitor of telomerase activity $[9,10]$. 
Recent studies have demonstrated that alternative splicing is an important gene regulation mechanism, helping to increase the diversity of proteins by favoring the production of large numbers of isoforms with dominant positive or negative functions [11]. However, almost one third of all alternative transcripts harbor a premature termination codon (PTC) and most are thought to be degraded by the nonsense-mediated mRNA decay (NMD) pathway [12].

NMD occurs in all eukaryotic cells. This cell surveillance system detects and rapidly degrades aberrant mRNAs containing PTCs (for reviews, see $[13,14]$ ). Termination codons are generally considered premature if they occur more than 50 to 55 nucleotides upstream from a final splice site recognized by the exon junction complex (EJC). The EJC serves as a platform for the binding of UPF factors, which are considered to be the conserved core of the NMD machinery. UPF1 is an ATP-dependent RNA helicase and RNA-dependent ATPase activated by phosphorylation. It is a crucial element of the NMD machinery, because silencing of the upf1 gene results in the stabilization of PTC-containing mRNAs in all organisms in which NMD has been investigated. This surveillance mechanism not only eliminates abnormal transcripts, but also controls transcript levels through a global system known as RUST (regulated unproductive splicing and translation) $[15,16]$. Several recent studies have demonstrated that the role of alternative splicing in gene regulation has been largely underestimated and have shown that this process is involved in homeostatic regulation, pathogenesis [17] and, particularly, in cancers $[18,19]$.

Gallid herpesvirus 2 (GaHV-2), also known as Marek's Disease Virus (MDV), is an avian oncogenic alphaherpesvirus that induces $\mathrm{T}$-cell lymphomas in chickens. These tumors develop within six weeks of infection in birds, making this model a unique asset for studies of the kinetics of disease induction and progression in a natural host system. This model has already proved valuable for studies of human lymphoma [20]. Indeed, Marek's disease is the only naturally occurring model for human lymphomas involving the overexpression of CD30 [21]. GaHV-2 is also the only virus harboring a viral homolog of the telomerase RNA template (vTR), with a sequence $88 \%$ identical to that of the chicken telomerase RNA (chTR). Functional analysis has shown that vTR can reconstitute telomerase activity by interacting with chicken TERT (chTERT) more efficiently than chTR [22]. Furthermore, vTR expression increases during GaHV-2 lymphomagenesis and this increase is correlated with the upregulation of telomerase activity, which is not associated with an upregulation of chTERT transcription [23,24]. Telomerase is also activated by a number of human oncogenic viruses [25], but investigations in this case are obviously restricted to in vitro analyses of cell lines derived from tumors or transformed in vitro. The chicken telomerase has a number of key features in common with the human enzyme, in terms of the organization of the TERT and TR genes and the regulation of telomerase activity [26]. Furthermore, as for hTERT, many alternative variants of chTERT have been identified and are thought to play a role in the regulation of telomerase activity [26,27]

In this study, we investigated the alternative splicing of chTERT in the MDV lymphomagenesis model. We report the identification of 27 alternative transcripts of chicken TERT cloned during a comparison of three different chicken cell lines including MSB-1, a MDV tumor cell line. Five alternative transcripts were inframe transcripts, whereas the other 22 were found to harbor a PTC. We then demonstrated that the in-frame transcripts generated proteins with no telomerase activity in vitro, whereas telomerase activity was detected for the products of constitutively spliced transcripts. The major PTC-containing variant found in the MDV cell line was shown to be a target of NMD in an upf1 silencing test. Finally, investigation of the splicing-mediated regulation of chTERT in vivo, in MDV-infected chickens, demonstrated that basal telomerase activity in normal $\mathrm{T}$ cells was associated with a high ratio of in-frame non-functional isoforms to functional constitutively spliced chTERT. During the upregulation of telomerase associated with lymphomagenesis, an increase in active constitutively spliced chTERT transcript levels was observed that coincided with a switch in alternative splicing from the in-frame type to the NMD type. These results suggest that the regulation of telomerase activity is partly dependent on the fine regulation of splicing of chTERT, regulating the abundance of functional chTERT mRNA.

\section{Results}

\section{Complex profile of chTERT alternative transcripts}

We first compared the pattern of alternative chTERT transcript production from the MDV T- MSB-1 cell line with that of two other cell lines: the DT40 B cell line, and the epithelial LMH cell line. Three cDNA libraries encompassing the $\mathrm{T}$ and $\mathrm{RT}$ regions of chTERT were generated (Figure 1).

The ratio of constitutively to alternatively spliced TERT transcripts was the first major difference identified between cell lines. Constitutively spliced transcripts accounted for about half of all transcripts in both lymphoid cell lines $(52.4 \%$ and $47.6 \%$ for MSB1 and DT40, respectively), but only $24.4 \%$ of transcripts in the $\mathrm{LMH}$ cell line. However, no significant differences in telomerase activity were observed between the three cell lines (telomerase activity of 152272 for MSB1, 165818 for 


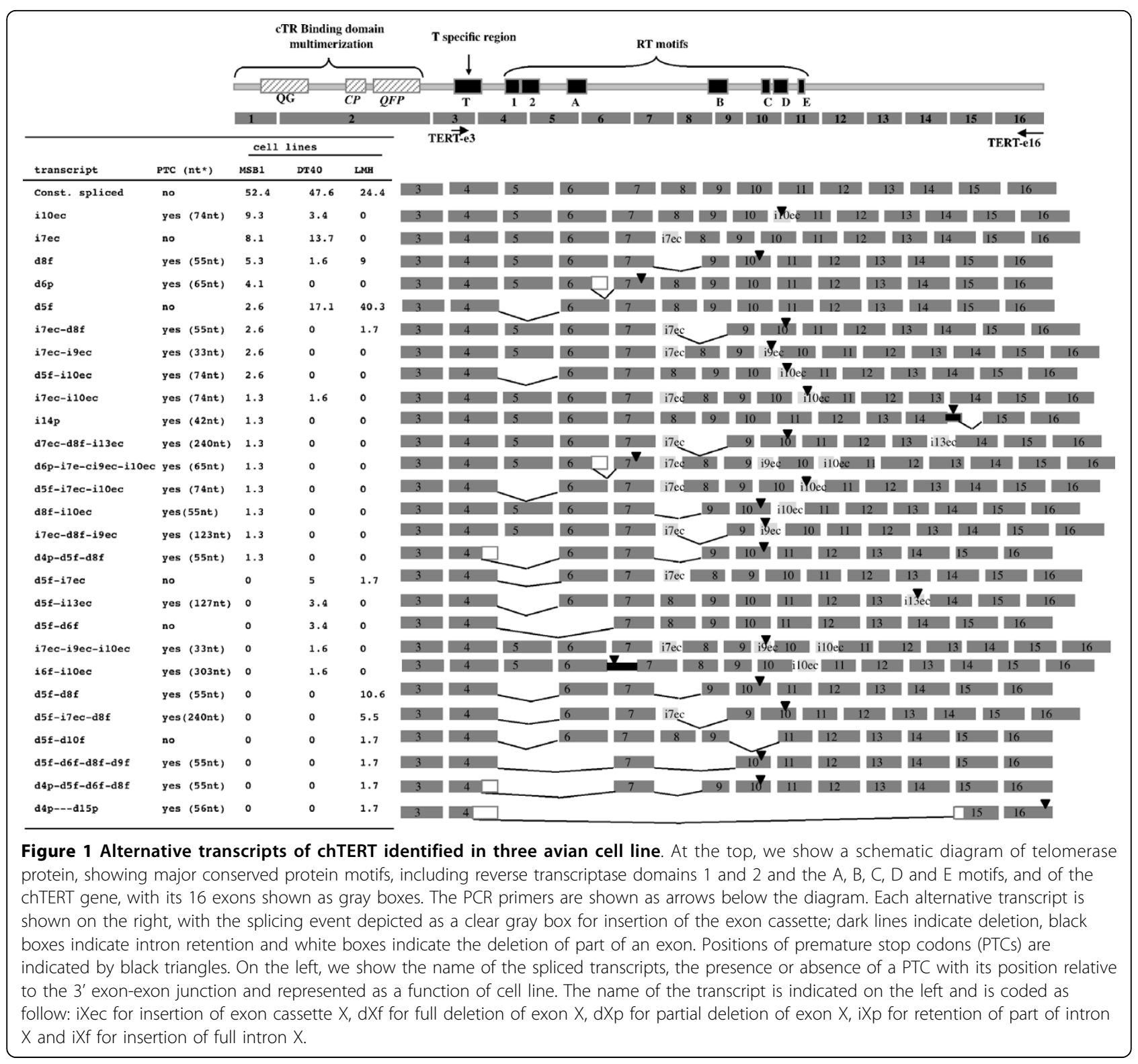

DT40 and 151115 for LMH) and there was therefore no clear correlation between this ratio and telomerase activity. In addition to constitutively spliced chTERT, we identified 27 alternative transcripts generated through the five typical splicing events: exon skipping, inclusion of a new exon cassette, cases of intron retention, use of alternative splice donor sites and use of alternative splice acceptor sites. Most of the alternative transcripts were generated by one of the four frequent splicing events: i7ec, i10ec, d5f and d8f. Each cell line displayed a number of rare specific transcripts generated by a specific combination of the four major events or a combination of one or more of these events with a rare splicing event (Figure 1).
The LMH cell line displayed a preferential deletion of exons, (Figure 1). By contrast, lymphoid cell lines were characterized by the insertion of introns generated by exonization (inclusion of intronic sequences in constitutive transcripts), through the formation of exon cassettes or the retention of all or part of an intron (Figure 1). Nevertheless, we observed differences in the distribution of splicing events between MSB1 T cells and DT40 B cells. For instance, major differences were seen for one of the major splicing events, i10ec (alone or in association with other splicing events), which was more frequently detected in MSB-1 than in DT40 cells (28.3\% versus $8.2 \%)$.

Most of the detected alternative transcripts (22/27) harbored premature termination codons (PTCs) (Figure 
1). Nineteen of these 22 transcripts harbored a PTC located more than 50 nucleotides upstream from the 3' splice junction and were predicted to result in NMDsensitive transcripts. Of the five remaining transcripts encoding in-frame isoforms of chTERT, only the i7ec transcript, corresponding to an inclusion of 12 amino acids (aa) between regions $A$ and $B$ of the protein, preserved the catalytic site of TERT, the four remaining transcripts involving the deletion of exon 5 (d5f), resulting in the deletion of amino acids from motifs 2 and $\mathrm{A}$ (Figure 1 and 2).

\section{None of the in-frame isoforms of chTERT is functional}

We assessed the telomerase activity of three reconstituted in-frame isoforms (Figure 2). Isoforms i7ec and $\mathrm{d} 5 \mathrm{f}$ were identified as alternative transcripts (Figure 1) and isoform $\mathrm{d} 10 \mathrm{f}$ was reconstructed for individual testing of the effect of the d10f splicing event, on which we subsequently focused specifically. We inserted the cDNAs of the isoforms downstream from the T7 promoter, for the assessment of telomerase activity in an in vitro assay [28]. Telomerase activity was measured in the presence of in vitro transcribed vTR or chTR. Consistent with published results [28], the full-length chTERT transcript reconstituted an efficient telomerase complex with both TRs with vTR that was $58 \%$ more efficient than chTR, whereas none of the in-frame spliced isoforms reconstituted telomerase activity. Thus, i7ec, despite the preservation of its RT motifs, displayed no telomerase activity and, as expected, in-frame isoforms with deletions in the RT domains were not able to reconstitute telomerase activity. As exon 5 (d5f) was deleted from all the four remaining in-frame isoforms other than i7ec, (Figure 1), we can conclude that all the in-frame isoforms are inactive. We then assessed the dominant negative function of these isoforms on telomerase activity when produced together with the active full-length chTERT (Figure 2C). All the isoforms acted as negative regulators, with inhibition levels of $74 \%$ for $\mathrm{d} 5 \mathrm{f}$ to $58 \%$ for $\mathrm{d} 10 \mathrm{f}$ when compared with the exclusive

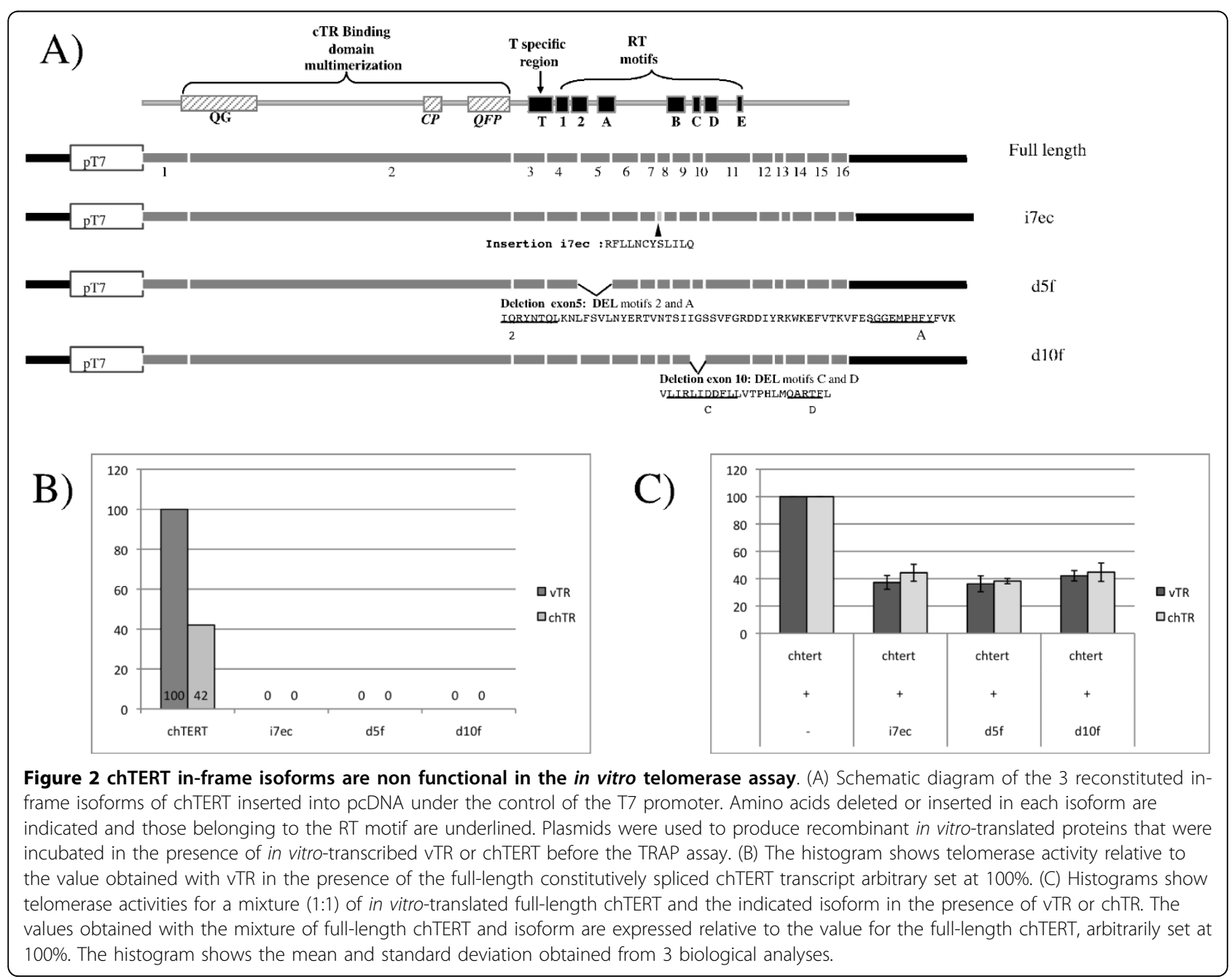


expression of full-length chTERT with vTR. No significant difference was seen between chTR and vTR, demonstrating that the inhibition mechanism did not depend on the nucleotide differences identified between vTR and chTR [28]. Thus, all the chTERT isoforms tested seemed to have dominant negative effects on telomerase activity, whether with chTR or vTR.

\section{The i10ec variant undergoes NMD}

The i10ec transcript was the most frequently represented transcript with predicted sensitivity to NMD in the MSB1 cell line. We therefore followed this transcript during lymphomagenesis. We evaluated its sensitivity to NMD by blocking the NMD pathway, using siRNA to target UPF1, the major NMD factor in the transfected LMH cell line. We chose to study LMH cells for technical reasons, essentially linked to transfection efficiency. Indeed MSB1 (or DT40) cells are lymphocytes and are difficult to transfect (2 to $5 \%$ transfection efficiency associated with high mortality rates of 70-90\%). As i10ec was not detected in chTERT alternative transcripts of $\mathrm{LMH}$ (Figure 1), we first determined whether transcripts harboring the i10ec splice event and thus defined as variant i10ec were detectable by PCR on LMH cDNA followed by fragment capillary electrophoresis analysis assays (CEAA) (see additional file 1: comparison of cDNA library and fragment analysis). This more sensitive technique, focusing on the specific amplification, by PCR, of short fragments, led to detection of the i10ec variant at a frequency of 1 to $5 \%$ with respect to full- length chTERT, thus rendering silencing experiments feasible. We observed significant increases in relative i10ec levels of 1.6 and 1.7 when the NMD pathway was inhibited with 25 and 50 pmol of siRNA against UPF1, respectively, as shown by comparisons with a nonsilenced sample used as a control and normalized to 1 (Figure 3A). The effective targeting of UPF1 by siRNA was also confirmed by monitoring UPF1 mRNA levels. UPF1 was strongly downregulated by 25 and 50 pmol of siRNA UPF1 (71 and 81\%, respectively) (Figure 3B). These results demonstrate that the $\mathrm{i} 10 \mathrm{ec}$ variant was efficiently detected and degraded by NMD.

\section{The increase in telomerase activity during the} lymphomagenesis induced by MDV is related to the upregulation of constitutively spliced ChTERT at the expense of alternatively spliced in-frame isoforms

Finally, we investigated the contribution of chTERT alternative splicing to the regulation of telomerase activity during the course of the lymphomagenesis induced by MDV. As it is difficult to amplify cDNA from the relatively long chTERT mRNA fragment present at low abundance in PBLs, we developed two independent fragment capillary electrophoresis analysis assays for investigating the dynamics of chTERT splicing regulation in vivo. In our assay, we chose to monitor changes in the ratio of the constitutively spliced transcript to variants resulting from two major splicing events, d5f and i10ec, identified in the MSB1 cell line, currently used as the reference cell line in investigations of Marek's disease

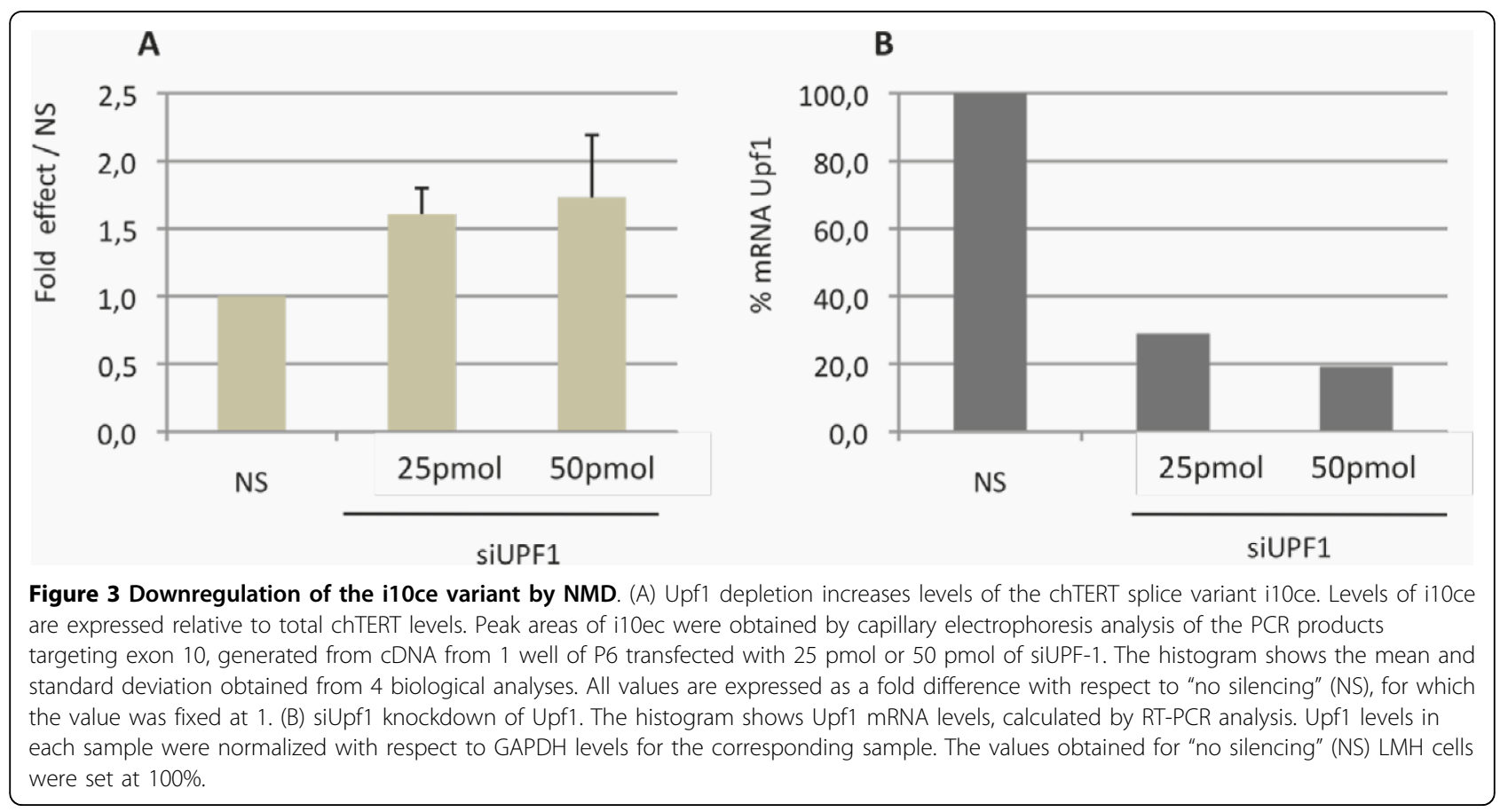




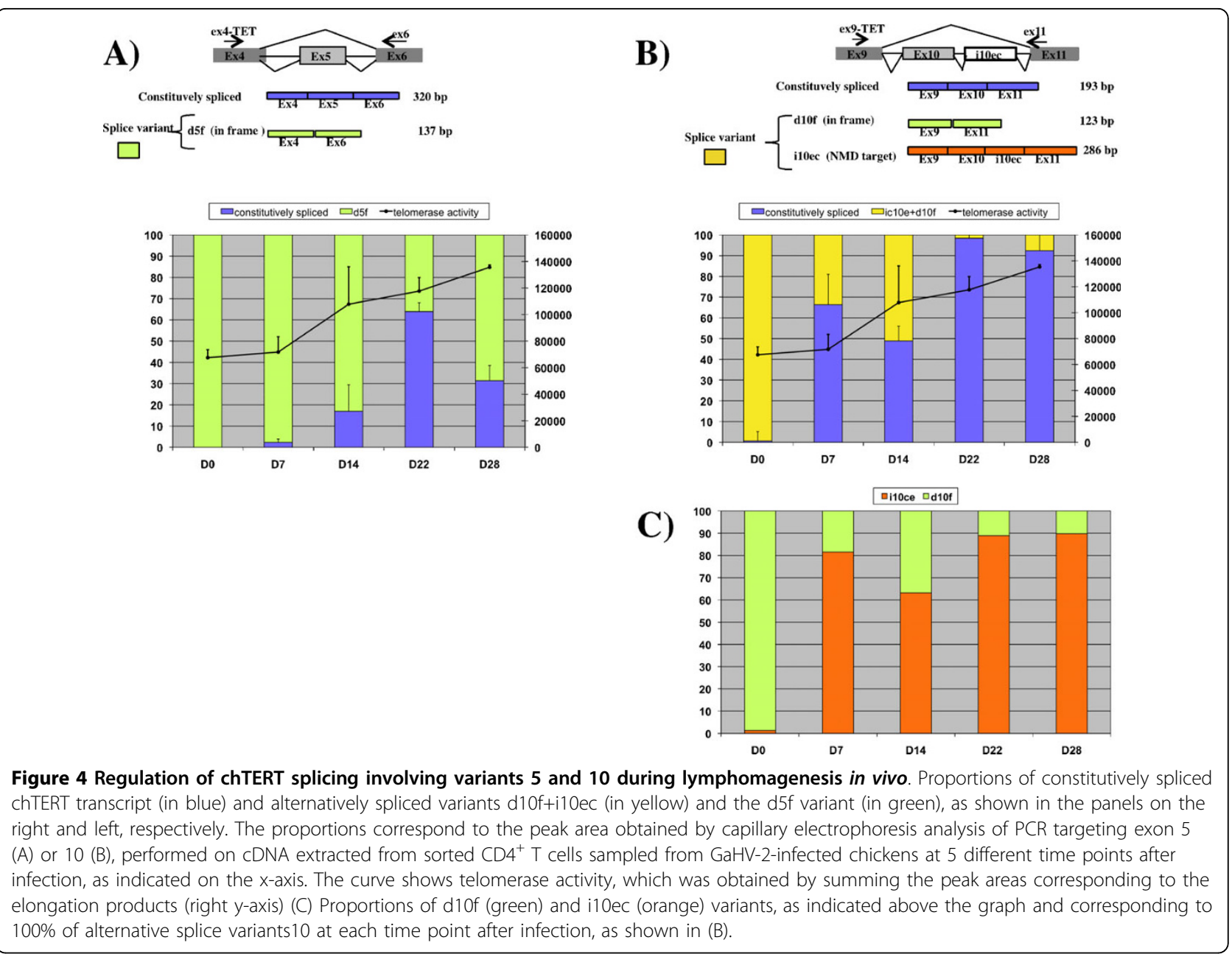

lymphoma and displaying some of the characteristics of lymphoma cells in vivo. Variant $\mathrm{d} 5 \mathrm{f}$ encodes a non-functional isoform of chTERT and i10ec, generating a variant targeted by the NMD pathway. Furthermore, the assay detecting the i10ec variant also detected the d10f variant, which would also be expected to generate a non-functional isoform, as demonstrated by the in vitro telomerase assay (Figure 2). Our assays were first optimized with the CD4+-sorted MSB1 cell line. The results obtained in fragment capillary electrophoresis analysis assays of cDNA samples were similar to those obtained for library analysis, although the ratios differed very slightly (see additional file 1: comparison of cDNA library and fragment analysis). These results confirm that fragment analysis is an appropriate technique for studying spliced chTERT and is suitable for use with sorted chicken $\mathrm{CD}^{+} \mathrm{T}$ cells.

We first measured telomerase activity at five previously identified crucial time points (d0, d7, d14, d22 and d28) [24](Figure 4). The results obtained with $\mathrm{CD}^{+} \mathrm{T}$ cells were similar to those obtained with PBL: (i) basal telomerase activity was detected in $\mathrm{CD}^{+} \mathrm{T}$ cells from non infected chickens (67527), (ii) a slight induction of telomerase activity was first observed at 7 days (71750), corresponding to the phase of primary semi-productive infection (iii), this induction peaked at 14 days p.i. (107800), corresponding to the expected time of lymphoma onset and (iv) persisted until 28 days p.i. (135310). Capillary electrophoresis analysis of splicing variants 5 and 10 was then performed on cDNA extracted from the same CD4 ${ }^{+} \mathrm{T}$-cell samples. Variants 5 and 10 had three major features in common: i) almost all the chTERT transcripts detected in CD4+ T cells from non infected chickens in tests for variants 5 and 10 were alternatively spliced ( $99.8 \%$ and $99.3 \%$ respectively), ii) in detection tests for both these variants, constitutively spliced transcripts became detectable from day 7 p.i., and their detection coincided with the increase in telomerase activity, iii) constitutively spliced transcript levels increased throughout infection (Figure 4).

However, the changes in splicing pattern differed between variants 5 and 10 . For the $\mathrm{d} 5 \mathrm{f}$ variant, we 
observed a progressive increase in the levels of constitutively spliced transcripts from day 7 (2.3\%), peaking at d22 (63.9\%) (Figure 4A). By contrast, with variant 10 (encompassing both d10f and i10ec), the levels of constitutively spliced transcript increased considerably between $\mathrm{d} 0$ and $\mathrm{d} 7$ (from $0.7 \%$ to $66.4 \%$ ) and remained high until day 28 (92.4\%) (Figure 4B). Investigation of the ratio of $\mathrm{d} 10 \mathrm{f}$ to i10ec showed a clear shift from $\mathrm{d} 10 \mathrm{f}$ to i10ec at $\mathrm{d} 7$ (Figure $4 \mathrm{C}$ ). Thus, at d0, the d10f variant, was, with $\mathrm{d} 5 \mathrm{f}$, almost the only alternatively spliced variant detectable. Then, beginning on $\mathrm{d} 7$, the proportion of the i10ec variant, which was predicted to be sensitive to NMD, increased strongly ( $>60 \%)$. These results indicate that (i) variants encoding in-frame non functional isoforms ( $\mathrm{d} 5 \mathrm{f}$ and $\mathrm{d} 10 \mathrm{f}$ ) predominate in uninfected CD4 ${ }^{+} \mathrm{T}$ cells and are associated with basal levels of telomerase activity (ii) the activation of telomerase is accompanied by an increase in the proportion of constitutively spliced transcripts and a decrease in the proportion of in-frame alternatively spliced transcripts, favoring the predicted NMD-sensitive transcript.

\section{Discussion}

The regulation of telomerase activity is a complex process involving several steps operating at both the transcriptional and post-transcriptional levels. In recent years, TERT splicing has been extensively studied in many types of human cells, tissues and tumors and in other organisms, such as plants, nematodes and ciliates [29]. A number of different alternative transcripts have been described, but little is known about the cause of splicing variant production and the ways in which splicing variant levels are regulated [29]. This is particularly true for the regulation of splicing during the cell transformation process and the dynamics of this process in vivo. In this study, we carried out RT-PCR fragment analysis to study the dynamics of the ratio of the constitutively spliced productive form of chTERT to three alternatively spliced non productive forms - one NMDsensitive transcript and two in-frame transcripts - in chicken $\mathrm{CD}^{+} \mathrm{T}$ cells, during a lymphomagenic process induced by MDV, an oncogenic herpes virus, in its natural host. In our model, lymphoma was observed on day 28 p.i. in $100 \%$ of infected chickens, facilitating analyses of the dynamics of gene regulation during oncogenesis in vivo [24]. Another major and unique advantage of our model is that we were able to control the start of tumorigenesis through the inoculation of chickens with the virus. Using this approach, we have previously shown that telomerase activation begins seven days after infection and peaks 22 days after infection, this time point being correlated with the first detection of the tumor in animals. Furthermore, telomerase activation and lymphomagenesis were correlated with upregulation of the vTR transcript, but no upregulation of the chTR and chTERT transcripts was seen $[23,24]$. In this study, we investigated telomerase regulation in more detail, by following the regulation of chTERT splicing in this model. Constitutively spliced transcript was almost undetectable at $\mathrm{d} 0$ in $\mathrm{CD}^{+} \mathrm{T}$ lymphocytes, but its levels gradually increased, peaking 22 days after infection, on the day of lymphoma onset. These data are the first to be obtained in vivo and are consistent with previous in vitro observations after the $v$-Rel transformation of spleen cells from chickens [27]. Moreover, the regulation of telomerase activity in human $\mathrm{T}$ lymphocytes seems to be similar, as full-length hTERT is undetectable in normal $\mathrm{T}$ cells and an increase in telomerase activity in activated $\mathrm{T}$ cells is associated with the induction of full-length hTERT mRNA production [30]. We also confirmed the results obtained for in vitro analyses of human tissues, with telomerase activation involving the induction, or a switch to production of the fulllength hTERT mRNA, as shown in various tissues [31-34].

Studies on spliced hTERT transcripts have generally focused on two major isoforms of hTERT: alpha (inframe isoform with a 36 bp deletion in motif A) and beta (isoform introducing a PTC in exon 10) [35]. The in-frame alpha isoform of hTERT has been shown to regulate telomerase activity negatively, acting as a dominant negative inhibitor $[9,10]$. We identified five inframe isoforms of chTERT, all of which had modifications affecting the RT motifs of telomerase. We have shown that three reconstituted in-frame isoforms, i7ec, $\mathrm{d} 5 \mathrm{f}$ and $\mathrm{d} 10 \mathrm{f}$, are inactive, being unable to reconstitute telomerase activity in vitro with either vTR or chTR. These isoforms also seem to act as negative regulators, as the association of the full-length chTERT transcript with an in-frame isoform decreased telomerase activity by a factor of 2 to 2.5 in the in vitro telomerase assay (Figure 2C). We can therefore hypothesize that the low basal levels of telomerase activity in normal $\mathrm{T}$ lymphocytes are controlled by in-frame non-functional isoforms, as these are almost the only isoforms detected (>99\%). The basal telomerase activity observed at d0 (Figure 4) was associated with very low levels of constitutively spliced active chTERT transcripts $(0.5 \%)$, consistent with a previous study [36] demonstrating that telomerase-positive cell lines contain only a few molecules of functional hTERT mRNA.

We also observed that telomerase activation associated with an increase in constitutively spliced transcript levels was accompanied by a switch in the profile of alternative transcripts from in-frame transcripts encoding non functional isoforms to NMD-sensitive transcripts. The ratio of the NMD-sensitive i10ec to the inframe d10f increased markedly with the upregulation of 


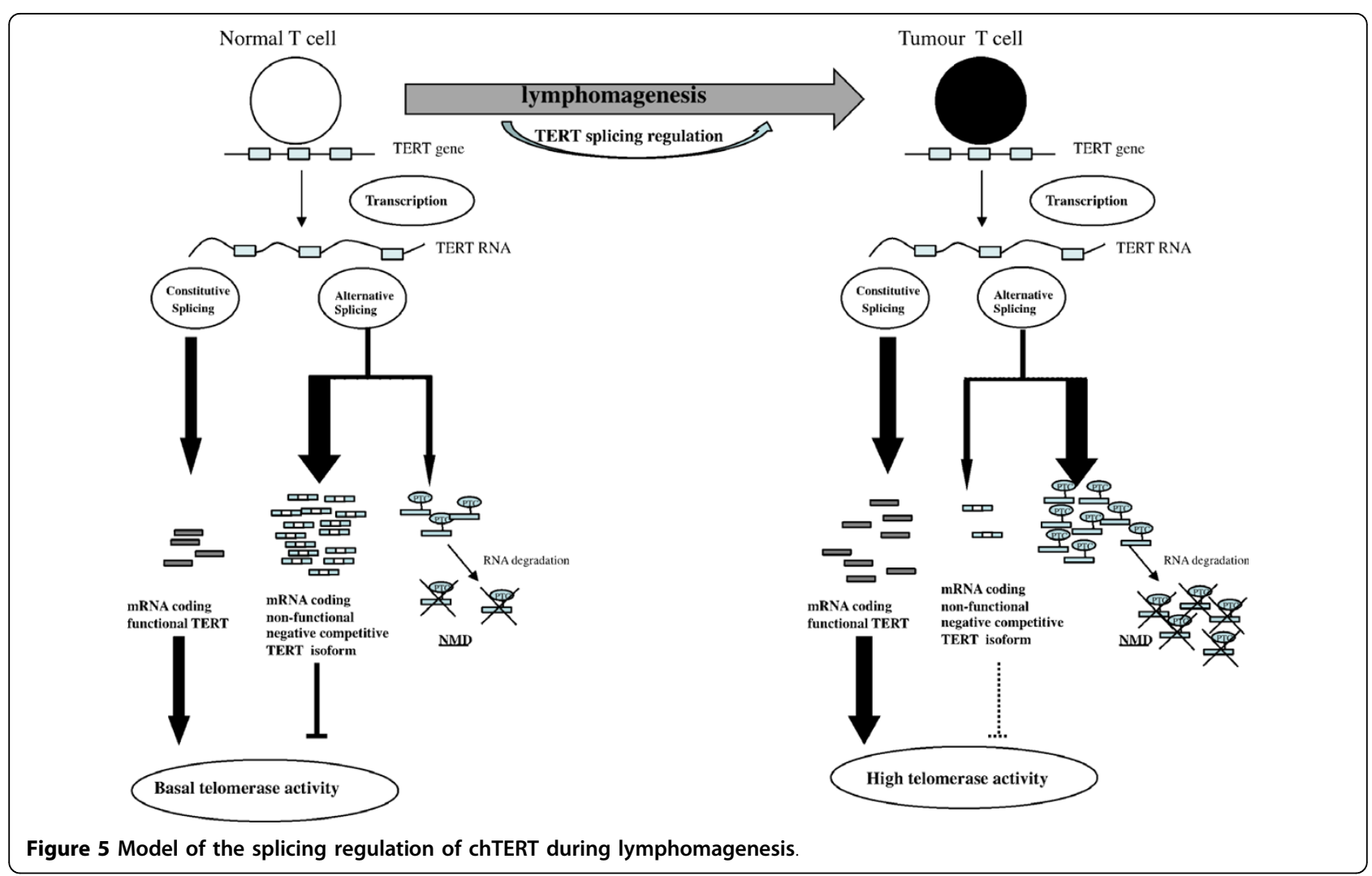

telomerase activity, from d7 onwards (Figure 4). The PTC of i10ec was located 74 nucleotides upstream from the last exon-exon junction, consistent with the definition of PTCs targeted by the NMD pathway. Nevertheless, the NMD sensitivity of the PTC-containing i10ec transcript was definitively confirmed by UPF1 silencing, which increased i10ec levels by a factor of up to 1.7 (Figure 3), consistent with previous reports of a increase in mRNA levels by a factor of 1.8 for PTC-containing COL alpha-2 [37] or H-ras [38]. The chTERT i10ec splice variant was thus identified as a new NMD target. These results confirm the hypothesis put forward by Chang H and Delany M. E. (2006) in a study identifying 19 chTERT spliced variants in different cell types, 16 of which (including i10ec) were predicted to have a PTC leading to degradation by the NMD pathway.

The change in the ratio of d10f to i10ec strongly suggests that differential splicing in favor of the NMD-sensitive transcript may be regulated by splicing activators or repressors, leading to the overrepresentation of constitutively spliced transcripts with respect to in-frame negative regulator isoforms. Recent studies have indicated that NMD is a sophisticated tool in physiological autoregulatory gene expression [12]. Lewis et al. [15] suggested that the coupling of splicing and NMD results in RUST, the mechanism regulating the ratio of productive and unproductive spliced forms of many genes, as demonstrated for the splicing factor SC35 [39]. Figure 5 schematically represents a model accounting for our observations. In lymphocytes, in which the ratio of constitutively spliced and in-frame negative regulator isoforms of TERT must be tightly controlled to maintain basal telomerase activity, the ratio of NMD-sensitive transcripts may be a major sensor (Figure 5). Moreover, with telomerase upregulation, the switch to NMD-sensitive transcripts may contribute to downregulation of the in-frame isoform acting as a negative regulator (Figure 5). Global regulation of this type, involving both an inframe isoform and an NMD-sensitive transcript, has been described for serum response factor (SRF) [40]. In this system, the abundance of SRF is reduced by RUST, but SRF gene expression is also regulated by an inframe isoform of SRF repressing transcription of the SRF gene itself. In our model, this feedback mechanism not only makes use of the active transcript ratio, but is also based on the negative regulator ratio. Interestingly, this model has also been proposed for regulation of the abundance of SR protein [41].

\section{Conclusion}

Our findings strongly suggest that the regulation of TERT splicing plays a key role in the upregulation of telomerase activity in vivo, by controlling the proportion of active constitutively spliced transcripts with an in- 
frame negative regulator and a third partner, an NMDsensitive transcript. Regulation of the ratio of NMD-sensitive transcript to in-frame transcript encoding the negative regulator isoform also plays a key role in suppressing telomerase activity in normal cells, in which negative regulator isoforms predominate, whereas NMD-sensitive transcripts become predominant when telomerase is upregulated during oncogenesis.

\section{Methods}

\section{Cell line culture and experimental assay in vivo}

Three avian cell lines were used: GaHV-2 lymphomaderived MSB-1 T cells [42] currently used as the reference cell line in Marek's disease investigation, the B-cell line DT40 [43] and the LMH hepatocellular carcinoma cell line [44]. Six-week-old White Leghorn specified pathogen-free susceptible B13B13 chickens were infected with GaHV-2 RB-1B virus [45]. Blood was obtained from two birds at 7, 14 and 22 days post-infection (dpi) and from one bird at $28 \mathrm{dpi}$, as the other bird had already died. The first time point ( $0 \mathrm{dpi})$, corresponded to the pooling of $2 \mathrm{ml}$ blood samples from each of the chickens, taken before infection. All experimental procedures were conducted in accordance with approved protocols for the use of animals in research.

\section{Transient transfection with siRNA}

Transient transfection with siRNA was carried out in six-well plates. LMH cells were transfected with 25 or 50 pmol of siRNA per well, in the presence of Lipofectamine 2000 reagent (Invitrogen), according to the manufacturer's protocol. Cells were harvested 48 hours later for RT-PCR analysis of UPF1, GAPDH and chTERT splicing variant i10ec. The siRNA targeting UPF1 (siUPF1) [GGUGGAAGAUGUAAUUAUU] was designed and purchased from Eurogentec.

\section{Flow cytometry analysis and cell sorting}

Peripheral blood lymphocytes (PBL) were isolated from blood [46] and immunolabeled for the sorting of $\mathrm{CD}^{+}$ $\mathrm{T}$ cells. The CD4 antigen was detected by incubation with the monoclonal antibody (Ab) CD4-UNLB, 821001 (Southern Biotechnology), followed by goat antimouse Ig conjugated to R-phycoerythrin (PE) (Jackson Immunoresearch). The cells were then analyzed by flow cytometry and PE-labeled cells were sorted and recovered for RNA and protein extraction, with a MoFlo ${ }^{\text {тM }}$ apparatus (Beckmann Coulter, Fort Collins, USA) and a high-speed cell sorter. The sorting speed was around 35,000 cells per second.

RNA extraction, CDNA synthesis and PCR amplification Total RNA was extracted from $2 \times 10^{6}$ cells, with the RNAble Kit (Eurobio). The cDNA was synthesized with
SuperScript III reverse transcriptase (Invitrogen), using oligo (dT) priming (Eurogentec), according to the manufacturer's instructions. The RT region of the chTERT gene, and gapdh and upf1 genes were amplified by PCR in Ready Master Mix 1.1X (Abgene), using three pairs of primers: TERT-e3 with TERT-e16 (Table 1, Figure 1A), GAPDHs (GTCCTCTCTGGCAAAGTCCAAG) with GAPDHr (CCACAACATACTCAGCACCTGC) designed from the Gallus gallus gapdh sequence (NCBI genebank number M11213.1) and UPF1s (GGGCT CCGAGTTCGAGTTCA) with UPF1r (CAGAATGCCATCCGGTCCAT) from the predicted sequence of the Gallus gallus upf1 gene (NCBI reference sequence NC_006115.2). The PCR products for UPF1 and GAPDH were analyzed by agarose gel electrophoresis. Adobe Photoshop 7.0 software (Adobe Systems, USA) was used to calculate the signal intensities for UPF1 relative to the GAPDH loading control.

\section{Generation and screening of cell-line cDNA libraries}

PCR products corresponding to the chTERT RT region were inserted into the pGEM-T Easy vector (Promega) and positive clones were randomly selected after screening for the presence of the insert by PCR. Individual selected clones were then screened for the type of alternative transcript, by running PCR with a panel of primer pairs (Table 1) covering each of the exons (4 to 15 ) of the chTERT cDNA. For the validation of exon PCR screening, 13 representative clones were also completely sequenced (MWG Biotech). Sequences were analyzed with DNASTAR sequence analysis software (Lasergene).

\section{PCR fragment analysis of chTERT splicing variants 5 and} 10

The ratios of constitutively to alternatively spliced transcripts from cell lines and sorted $\mathrm{CD} 4^{+} \mathrm{T}$ cells were determined by fluorescent fragment analysis [23,24]. Nested-PCR was performed on the first PCR amplification product of the chTERT RT region, in Ready Master Mix 1.1X (Abgene), with specific tetrachlorofluorescein phosphoramidite-labeled forward primers and unlabeled reverse primers specific for exon 5 or 10 (Table 1). Amplification products were analyzed with an automated ABI Prism 310 fragment analyzer (Perkin Elmer Life Sciences) and the ratio of spliced transcripts was determined as a percentage of the peak area for constitutively $(C)$ versus alternatively $(A)$ spliced transcripts. Each assay was performed at least three times.

\section{Reconstitution in vitro of the telomerase complex}

The three cDNAs for alternative transcripts of chTERT - d5f, d10f, and i10ec - were inserted into pcDNA 3 and the telomerase complex was reconstituted [28]. We incubated $1 \mu \mathrm{g}$ of $\mathrm{vTR}$ or chTR RNA transcribed in 
Table 1 Primers used in the study

\begin{tabular}{|c|c|c|c|}
\hline Oligonucleotide $^{1}$ & Localization $^{2}$ & Sequence $^{3}$ & Screening \\
\hline tert e3-M331s & e3 (2210-39) & CTGTTCCTGCCTATGAACATTGTTACCGTG & chTERT cDNA \\
\hline tert e3M137-s & e3 $(2260-80)$ & CTATACTGGCTGATGGATTCC & exon 4 \\
\hline M139s & e4 (2480-500) & CATCAAGGCTCCGGTTCATTC & exon 5 \\
\hline M141s & e5 (2680-700) & GGGAGAGATGATATCTACAGG & exon 6 \\
\hline M142r & e5 (2700-680) & CCTGTAGATATCATCTCTCCC & exon 4 \\
\hline M143s & e6 (2815-35) & GTGGAAGTGATATCACAGGTC & exon 7 \\
\hline M144r & e6 (2835-15) & GACCTGTGATATCACTTCCAC & exon 5 \\
\hline M145s & e7 (2974-92) & GTGTCCAAGCTTCAAGAGA & exon 8 \\
\hline M146r & e7 (2992-74) & TCTCTTGAAGCTTGGACAC & exon 6 \\
\hline M147s & e8 (3040-60) & AATGAGAACAGTTCCACCCTG & exon 9 \\
\hline M148r & e8 (3060-40) & CAGGGTGGAACTGTTCTCATT & exon 7 \\
\hline M149s & e9 (3172-92) & AGCTTATGCTACGGAGACATG & exon 10 \\
\hline M150r & e9 (3192-72) & CATGTCTCCGTAGCATAAGCT & exon 8 \\
\hline M151s & e10 (3258-79) & GCTGGTTACGCCACATTTAATG & exon 11 \\
\hline M152r & e10 (3279-58) & CATTAAATGTGGCGTAACCAGC & exon 9 \\
\hline M153s & e11 (3373-93) & GATGATATCCCGGGATGTTCC & exon 12 \\
\hline M154r & e11 (3393-73) & GGAACATCCCGGGATATCATC & exon 10 \\
\hline M155s & e12 (3556-76) & TGCAAATTGACTGCAGTCCTC & exon 13 \\
\hline rM156r & e12 (3576-56) & GAGGACTGCAGTCAATTTGCA & exon 11 \\
\hline M157s & e13 (3618-42) & CAGCCTTCAGACAGTTCTAATTAAC & exon 14 \\
\hline M158r & e13 (3642-18) & GTTAATTAGAACTGTCTGAAGGCT & exon 12 \\
\hline M159s & e14 (3727-47) & CCTGATTTCTTCCTAAGGATC & exon 15 \\
\hline M160r & e14 (3747-27) & GATCCTTAGGAAGAAATCAGG & exon 13 \\
\hline M162r & e15 (3870-50) & ATGGTAGCACAGCCATTCTGC & exon 14 \\
\hline M163r & e16 (3996-76) & CACCGTCTTCAGCAGTTCCAT & exon 15 \\
\hline tert e16-M316-r & e16 (4041-07) & TTAGTCCAGTATAGTITTGAAATCTTGACAAAGCG & ChTERT CDNA \\
\hline TET-tert e4 & e4 (2554-78) & TET-CAGAAACTCAGCAAGGAAAGCAGAG & splice variant 5 \\
\hline tert e6 & e6 (2874-55) & CCACCTTATTCCATAGACAG & splice variant 5 \\
\hline TET-tert e9 & e9 (3170-92) & TET-GCAGCTTATGCTACGGAGACATG & splice variant 10 \\
\hline tert e11 & e11 (3361-42) & TCACCACAGTCTTCTTGGCA & splice variant 10 \\
\hline
\end{tabular}

${ }^{1}$ name of the primer with TET labeling indication,

${ }^{2}$ localization in the chTERT cDNA sequence AY626231 (NCBI n ${ }^{\circ}$ )

${ }^{3}$ written in the $5^{\prime}->3^{\prime}$ orientation

vitro with T7 polymerase, and $2 \mu \mathrm{l}$ of alternatively spliced and full-length chTERT transcripts expressed in the TNT system (Promega). We assessed the inhibition of telomerase activity by the isoforms with $2 \mu \mathrm{l}$ of the in vitro-translated full-length isoform and $2 \mu \mathrm{l}$ of in vitrotranslated isoform. Aliquots of ribonucleoprotein assembly products were used for the assessment of telomerase activity in the telomere repeat amplification protocol (TRAP) assay [28].

\section{Telomere repeat amplification protocol (TRAP) assay}

The telomerase activity of sorted $\mathrm{CD} 4^{+} \mathrm{T}$ cells from chicken in in vivo infection assays and of in vitro reconstituted complexes was quantified as previously described $[28,24]$. Briefly, telomerase activity was quantified with $500 \mathrm{ng}$ of protein extracted from CD4 $+\mathrm{T}$ cells [24] or $0.3 \mu \mathrm{l}$ of in vitro ribonucleoprotein assembly products [28]. PCR was carried out with the 6-carboxytetramethylrhodamine-labeled forward primer TS (5'-AATCCGTGCAGCAGAGTT-3') and CXext (5'-CCCTAACCCTAACCCTAACCCTAA-3') as the reverse primer. An internal amplification standard (ITAS) was added to the PCR mixture in quantitative TRAP assays or was used independently as a PCR control. PCR products were analyzed by capillary electrophoresis (ABI Prism 310; PerKinElmer Life Sciences). The telomerase activity of each protein extract was estimated by adding together the integrated values for each telomerase elongation product of at least $60 \mathrm{bp}$. 


\section{Additional material}

Additional file 1: Comparison of estimates of the levels of splicing variants 5 and 10 by cDNA cloning and fragment electrophoresis. Proportions of constitutively spliced chTERT transcript (in blue) and alternatively spliced variants d10f (in green), 10ec (in orange) and d5f (in green). The proportions correspond to the peak area obtained by capillary electrophoresis analysis (CEAA) of PCR targeting variant 10 (A) or 5 (B), performed on cDNA extracted from LMH or MSB1 cells (3 biological analyses) or obtained from CDNA analysis by estimation of the percentage of all alternative transcripts harboring splicing event 5 or 10 (Figure 1).

\section{Abbreviations}

TERT: telomerase reverse transcriptase; TR: telomerase RNA; NMD: nonsensemediated decay; PTC: premature termination codon, TRAP: telomere repeat amplification protocol

\section{Acknowledgements}

We would like to thank Laurence Mérat, Bruno Campone, Patrice Cousin and Jean Méry for taking care of the chickens used in the experiments. We also thank Dominique Kerboeuf for constructive discussions about flow cytometry analysis and Emmanuel Kut, Laetitia Fragnet, Gregory Douard, Camille and Perrine Rasschaert for their help with the cloning of the alternatively spliced chTERT transcripts, telomerase activity quantification and the screening of cDNA libraries. S. Amor held a PhD grant from Région Centre (France). This work was supported by grants from the Ligue contre le Cancer - Comités du Cher, de l'Indre, de l'Eure (France).

\section{Author details}

${ }^{1}$ Equipe TLVI, Université François Rabelais de Tours, UFR Sciences et Techniques, Parc de Grandmont 37200 Tours France. ${ }^{2}$ INRA-Département de Santé Animale - Centre de recherches de Tours - 37380 Nouzilly - France. ${ }^{3}$ Centre de recherches INRA de Tours-UR IASP - Service de Cytométrie 37380 Nouzilly-France.

\section{Authors' contributions}

Study concept and design: SL and DR; Acquisition of data SA, SR, YLV, GD and SL; Analysis and interpretation of data: SA, SL, GD and DR; Drafting of manuscript: SA, SL; Critical revision of manuscript SL, GD and DR: Obtainment of funding: SL, GD and DR; Study supervision SL, DR. All authors have read and approved the final manuscript.

\section{Competing interests}

The authors declare that they have no competing interests.

Received: 3 June 2010 Accepted: 21 October 2010

Published: 21 October 2010

\section{References}

1. Greider CW, Blackburn EH: Identification of a specific telomere terminal transferase activity in Tetrahymena extracts. Cell 1985, 43(2 Pt 1):405-413.

2. Dong CK, Masutomi K, Hahn WC: Telomerase: regulation, function and transformation. Crit Rev Oncol Hematol 2005, 54(2):85-93.

3. Kim NW, Piatyszek MA, Prowse KR, Harley CB, West MD, Ho PL, Coviello GM, Wright WE, Weinrich SL, Shay JW: Specific association of human telomerase activity with immortal cells and cancer. Science 1994, 266(5193):2011-2015.

4. Janknecht R: On the road to immortality: hTERT upregulation in cancer cells. FEBS Lett 2004, 564(1-2):9-13.

5. Pan $\mathrm{C}$, Xue $\mathrm{BH}$, Ellis TM, Peace DJ, Diaz MO: Changes in telomerase activity and telomere length during human $T$ lymphocyte senescence. Exp Cell Res 1997, 231(2):346-353.

6. Feng J, Funk WD, Wang SS, Weinrich SL, Avilion AA, Chiu CP, Adams RR, Chang E, Allsopp RC, Yu J, et al: The RNA component of human telomerase. Science 1995, 269(5228):1236-1241.
7. Cong YS, Wright WE, Shay JW: Human telomerase and its regulation. Microbiol Mol Biol Rev 2002, 66(3):407-425, table of contents.

8. Saeboe-Larssen S, Fossberg E, Gaudernack G: Characterization of novel alternative splicing sites in human telomerase reverse transcriptase (hTERT): analysis of expression and mutual correlation in mRNA isoforms from normal and tumour tissues. BMC Mol Biol 2006, 7:26.

9. Yi X, White DM, Aisner DL, Baur JA, Wright WE, Shay JW: An alternate splicing variant of the human telomerase catalytic subunit inhibits telomerase activity. Neoplasia 2000, 2(5):433-440.

10. Colgin LM, Wilkinson C, Englezou A, Kilian A, Robinson MO, Reddel RR: The hTERTalpha splice variant is a dominant negative inhibitor of telomerase activity. Neoplasia 2000, 2(5):426-432.

11. Kim E, Magen A, Ast G: Different levels of alternative splicing among eukaryotes. Nucleic Acids Res 2007, 35(1):125-131.

12. Muhlemann $O$, Eberle $A B$, Stalder $L$, Zamudio Orozco R: Recognition and elimination of nonsense mRNA. Biochim Biophys Acta 2008, 1779(9):538-549.

13. Rehwinkel J, Raes J, Izaurralde E: Nonsense-mediated mRNA decay: Target genes and functional diversification of effectors. Trends Biochem Sci 2006, 31(11):639-646.

14. Silva $A L$, Romao $L$ : The mammalian nonsense-mediated mRNA decay pathway: to decay or not to decay! Which players make the decision? FEBS Lett 2009, 583(3):499-505.

15. Lewis BP, Green RE, Brenner SE: Evidence for the widespread coupling of alternative splicing and nonsense-mediated mRNA decay in humans. Proc Natl Acad Sci USA 2003, 100(1):189-192

16. Lareau LF, Brooks AN, Soergel DA, Meng Q, Brenner SE: The coupling of alternative splicing and nonsense-mediated mRNA decay. Adv Exp Med Biol 2007, 623:190-211.

17. Nissim-Rafinia $M$, Kerem $B$ : The splicing machinery is a genetic modifier of disease severity. Trends Genet 2005, 21(9):480-483.

18. Kim E, Goren A, Ast G: Insights into the connection between cancer and alternative splicing. Trends Genet 2008, 24(1):7-10.

19. Pettigrew CA, Brown MA: Pre-mRNA splicing aberrations and cancer Front Biosci 2008, 13:1090-1105

20. Osterrieder N, Kamil JP, Schumacher D, Tischer BK, Trapp S: Marek's disease virus: from miasma to model. Nat Rev Microbiol 2006, 4(4):283-294.

21. Burgess SC, Young JR, Baaten BJ, Hunt L, Ross LN, Parcells MS, Kumar PM, Tregaskes CA, Lee LF, Davison TF: Marek's disease is a natural model for lymphomas overexpressing Hodgkin's disease antigen (CD30). Proc Nat Acad Sci USA 2004, 101(38):13879-13884.

22. Fragnet $L$, Blasco MA, Klapper W, Rasschaert D: The RNA subunit of telomerase is encoded by Marek's disease virus. J Virol 2003, 77(10):5985-5996.

23. Shkreli M, Dambrine G, Soubieux D, Kut E, Rasschaert D: Involvement of the oncoprotein c-Myc in viral telomerase RNA gene regulation during Marek's disease virus-induced lymphomagenesis. J Virol 2007 81(9):4848-4857.

24. Debba-Pavard M, Ait-Lounis A, Soubieux D, Rasschaert D, Dambrine G: Vaccination against Marek's disease reduces telomerase activity and viral gene transcription in peripheral blood leukocytes from challenged chickens. Vaccine 2008, 26(38):4904-4912.

25. Bellon M, Nicot C: Regulation of telomerase and telomeres: human tumor viruses take control. J Natl Cancer Inst 2008, 100(2):98-108.

26. Chang H, Delany ME: Complicated RNA splicing of chicken telomerase reverse transcriptase revealed by profiling cells both positive and negative for telomerase activity. Gene 2006, 379:33-39.

27. Hrdlickova R, Nehyba J, Liss AS, Bose HR: Mechanism of telomerase activation by v-Rel and its contribution to transformation. J Virol 2006 , 80(1):281-295

28. Fragnet $L$, Kut $E$, Rasschaert D: Comparative functional study of the viral telomerase RNA based on natural mutations. J Biol Chem 2005, 280(25):23502-23515.

29. Sykorova E, Fajkus J: Structure-function relationships in telomerase genes. Biol Cell 2009, 101(7):375-392, 371 p following 392.

30. Jalink M, Ge Z, Liu C, Bjorkholm M, Gruber A, Xu D: Human normal T lymphocytes and lymphoid cell lines do express alternative splicing variants of human telomerase reverse transcriptase (hTERT) mRNA. Biochem Biophys Res Commun 2007, 353(4):999-1003

31. Fan $Y$, Liu Z, Fang $X$, Ge Z, Ge N, Jia Y, Sun $P$, Lou F, Bjorkholm $M$, Gruber $A$, et al: Differential expression of full-length telomerase reverse 
transcriptase mRNA and telomerase activity between normal and malignant renal tissues. Clin Cancer Res 2005, 11(12):4331-4337.

32. Villa R, Porta CD, Folini M, Daidone MG, Zaffaroni N: Possible regulation of telomerase activity by transcription and alternative splicing of telomerase reverse transcriptase in human melanoma. I Invest Dermatol 2001, 116(6):867-873

33. Lincz LF, Mudge LM, Scorgie FE, Sakoff JA, Hamilton CS, Seldon M: Quantification of hTERT splice variants in melanoma by SYBR green realtime polymerase chain reaction indicates a negative regulatory role for the beta deletion variant. Neoplasia 2008, 10(10):1131-1137.

34. Yokoyama Y, Wan X, Takahashi Y, Shinohara A, Tamaya T: Alternatively spliced variant deleting exons 7 and 8 of the human telomerase reverse transcriptase gene is dominantly expressed in the uterus. Mol Hum Reprod 2001, 7(9):853-857.

35. Kilian A, Bowtell DD, Abud HE, Hime GR, Venter DJ, Keese PK, Duncan EL, Reddel RR, Jefferson RA: Isolation of a candidate human telomerase catalytic subunit gene, which reveals complex splicing patterns in different cell types. Hum Mol Genet 1997, 6(12):2011-2019.

36. Yi X, Shay JW, Wright WE: Quantitation of telomerase components and hTERT mRNA splicing patterns in immortal human cells. Nucleic Acids Res 2001, 29(23):4818-4825.

37. Usuki F, Yamashita A, Kashima I, Higuchi I, Osame M, Ohno S: Specific inhibition of nonsense-mediated mRNA decay components, SMG-1 or Upf1, rescues the phenotype of Ullrich disease fibroblasts. Mol Ther 2006, 14(3):351-360.

38. Barbier J, Dutertre M, Bittencourt D, Sanchez G, Gratadou L, de la Grange P, Auboeuf D: Regulation of $\mathrm{H}$-ras splice variant expression by cross talk between the p53 and nonsense-mediated mRNA decay pathways. Mol Cell Biol 2007, 27(20):7315-7333.

39. Sureau A, Gattoni R, Dooghe Y, Stevenin J, Soret J: SC35 autoregulates its expression by promoting splicing events that destabilize its mRNAs. EMBO I 2001, 20(7):1785-1796.

40. Zhang X, Azhar G, Huang C, Cui C, Zhong Y, Huck S, Wei JY: Alternative splicing and nonsense-mediated mRNA decay regulate gene expression of serum response factor. Gene 2007, 400(1-2):131-139.

41. Palusa SG, Reddy AS: Extensive coupling of alternative splicing of premRNAs of serine/arginine (SR) genes with nonsense-mediated decay. New Phytol 2010, 185(1):83-89.

42. Akiyama $Y$, Kato $S$ : Two cell lines from lymphomas of Marek's disease. Biken J 1974, 17(3):105-116.

43. Baba TW, Giroir BP, Humphries EH: Cell lines derived from avian lymphomas exhibit two distinct phenotypes. Virology 1985, 144(1):139-151.

44. Kawaguchi T, Nomura K, Hirayama Y, Kitagawa T: Establishment and characterization of a chicken hepatocellular carcinoma cell line, LMH. Cancer Res 1987, 47(16):4460-4464.

45. Djeraba A, Bernardet N, Dambrine G, Quere P: Nitric oxide inhibits Marek's disease virus replication but is not the single decisive factor in interferon-gamma-mediated viral inhibition. Virology 2000, 277(1):58-65.

46. Djeraba-AitLounis A, Soubieux D, Klapper W, Rasschaert D: Induction of telomerase activity in avian lymphoblastoid cell line transformed by Marek's disease virus, MDCC-MSB1. Vet Pathol 2004, 41(4):405-407.

Pre-publication history

The pre-publication history for this paper can be accessed here: http://www.biomedcentral.com/1471-2407/10/571/prepub

doi:10.1186/1471-2407-10-571

Cite this article as: Amor et al:: Alternative splicing and nonsensemediated decay regulate telomerase reverse transcriptase (TERT) expression during virus-induced lymphomagenesis in vivo. BMC Cancer 2010 10:571.

\section{Submit your next manuscript to BioMed Central and take full advantage of:}

- Convenient online submission

- Thorough peer review

- No space constraints or color figure charges

- Immediate publication on acceptance

- Inclusion in PubMed, CAS, Scopus and Google Scholar

- Research which is freely available for redistribution

Submit your manuscript at www.biomedcentral.com/submit 